\title{
Finafloxacin: A Novel Fluoroquinolone Introduced in Clinical Trials
}

\section{Bela Kocsis* and Dora Szabo}

Institute of Medical Microbiology, Semmelweis University, Hungary

Quinolones were introduced into clinical practice in 1962, in form of nalidix acid and oxolinic acid and both were effective against most Enterobacteriaceae. Several changes on basic quinolone ring yielded fluoroquinolones that have increased antibacterial potency and broadened spectrum. Substituents on the basic chemical structure that improve pharmacokinetic properties are namely, fluorine atomin position 6; cyclopropyl or difluorophenyl in position 1; halogen, methoxy or fused third ring in position 8 ; and piperazine in position $7[1,2]$.

The most frequently applied fluoroquinolones in clinical use are ciprofloxacin, ofloxacin, levofloxacin, and moxifloxacin. Ciprofloxacin and ofloxacin are mostly used in treatment of urinary tract, intestinal infections caused by Gram-negative bacteria. Levofloxacin is an active isomer of ofloxacin and it possesses a broader-antibacterial spectrum, that includes Gram-negative as well as Gram-positive bacteria. Levofloxacin is mostly used in treatment of respiratory tract infections. Moxifloxacin's spectrum includes Gram-positive aerob and anaerob bacteria [3-5]. Fluoroquinolone mechanism of action is inhibition of bacterial DNA synthesis as these agents target bacterial DNA gyrase and topoisomerase IV enzymes [6-9]. Fluoroquinolone antibiotics have a concentration dependent antibacterial activity. Peak serum concentration is reached rapidly after administration, keeping its effect as long as the concentration exceeds the minimum inhibitory concentration (MIC) value [10].

Finafloxacin belongs to the class of fluoroquinolones with chemical structure including a 8-cyano-substituent and 7-pyrrolooxazinyl moiety. It has a zwitterion chemical structure with a $6.7 \mathrm{pH}$ isoelectric value. During in vitro studies finafloxacin showed extended spectrum activity against several human bacterial pathogens namely, methicillin-susceptible Staphylococcus aureus and methicillin-resistant Staphylococcus aureus, Streptococcus pneumoniae Legionella pneumophila, Listeria monocytogenes, Helicobacter pylori, uropathogenic bacteria including Escherichia coli, Pseudomonas aeruginosa, Klebsiella pneumoniae, Proteus mirabilis [11,12]. Finafloxacin can be administered in otic drop formulation for treating acute otitis externa infection and can be treatment option of both complicated and uncomplicated urinary tract infections. Its optimal bactericidal effect was noticed in acidic environment and Phase II studies have shown remarkable results about the oral administration of finafloxacin in combination with amoxicillin or omeprazol against H. pylori gastritis $[9,13-15]$.

The antibacterial effect of finafloxacin was investigated in infectious mouse models namely, in Burkholderia pseudomallei infection of BALB/c mice. Orally administrated finafloxacin $(37.5 \mathrm{mg} / \mathrm{kg}$ ) achieved the maximum concentration $(7.24 \mathrm{mg} / \mathrm{l})$ within 2 hours and resulted in eradication of the bacterium from lung, liver and spleen [16].

In vivo study demonstrated that $1 \mathrm{mg} / \mathrm{kg} /$ day dosage of oral finafloxacin lead to teratogenesis (neural tube and skeletal defects: spina bifida, missing lumbar vertebra and arch, sternebra fusion etc.) in rabbits [17].

Int J Clin Med Microbiol

ISSN: $2456-4028$

\section{Publication History:}

Received: October 23, 2018

Accepted: November 08, 2018

Published: November 10, 2018

\section{Keywords:}

Fluoroquinolone, Clinical trials, Toxicity

Finafloxacin is absorbed rapidly following oral administration and can be used both intravenously and locally. After 300-600-800 mg oral adminsitration of finafloxacin peak serum concentration (Cmax: 4.15 $\mathrm{mg} / \mathrm{l} ; 6.76 \mathrm{mg} / \mathrm{l} ; 8.95 \mathrm{mg} / \mathrm{l}$ ) was attained within 1 hour. Bioavability of oral formations possess $62-100 \%$, half life of about $10 \mathrm{~h}$ and prolonged post antibiotic effect was observed. Approximately $30 \%$ of a dose of finafloxacin is eliminated unchanged in the urine. Renal clearance was increased at dose of 100 and $200 \mathrm{mg}$ in contrast with higher dosage (400 and $800 \mathrm{mg}$ ) due to saturable transport mechanism $[18,19]$.

One study found that elimination rate was significantly lower in healthy eldery individuals, than healthy young volunteers. Urinary bactericidal concentration of finafloxacin was $69.3 \mathrm{mg} / \mathrm{ml}$ (given 200 $\mathrm{mg}$ single oral dose) and $150 \mathrm{mg} / \mathrm{l}$ (given $800 \mathrm{mg}$ single oral dose). In addition it was also observed that bactericidal effect of finafloxacin was enhanced against uropathogens such as E. coli, K. pneumoniae, $P$. mirabilis, P. aeruginosa and Enterococcus faecalis [19].

Finafloxacin exhibits higher activity in acidic urine, than in alkaline or neutral environment due to $\mathrm{pH}$ dependent function. It exhibits optimal activity at pH 5-6 in contrast with other fluoroquinolones that can't achieve antibiotic effect in acidic conditions. Finafloxacin achieves a 4 to 32-fold lower MIC against S.aureus and E. faecalis at pH 5.8 compared to ciprofloxacin MIC [11].

Furthermore, advanced activity was observed under anaerobic condition mainly at neutral $\mathrm{pH}$ value, that was verified by MIC values of finafloxacin against Gram-negative anaerob patogens including Bacteroides fragilis. It shows antibacterial effect with $0.5 / 4 \mu \mathrm{g} / \mathrm{ml}$ of $\mathrm{MIC}_{50} / \mathrm{MIC}_{90}$ at $\mathrm{pH} 7.2$ whilst these values were decreased $(0.25 / 4 \mu \mathrm{g} /$ $\mathrm{ml})$ under acidic conditions $[9,20]$.

Finafloxacin can pass into the cytoplasm of phagocytes thus it is adapted to kill the intracellular pathogens. Finafloxacin showed remarkable antibacterial effect against both $L$. monoytogenes and L. pneumophila. In comparison with ciprofloxacin the MIC values of finafloxacin (MIC of L. pneumophila $=0,01 \mathrm{mg} / \mathrm{l}$; MIC of $L$. monocytogenes $=1 \mathrm{mg} / \mathrm{l})$ for intracellular bacteria were similar than the other quinolones [14].

"Corresponding Author: Dr. Bela Kocsis, Institute of Medical Microbiology, Semmelweis University, Budapest, Üllöi út 26, 1085 Hungary; E-mail: kocsis.bela@med.semmelweis-univ.hu

Citation: Kocsis B, Szabo D (2018) Finafloxacin: A Novel Fluoroquinolone Introduced in Clinical Trials. Int J Clin Med Microbiol 3: 135. doi: https://doi. org/10.15344/2456-4028/2018/135

Copyright: (c) 2018 Kocsis et al. This is an open-access article distributed under the terms of the Creative Commons Attribution License, which permits unrestricted use, distribution, and reproduction in any medium, provided the original author and source are credited. 
Citation: Kocsis B, Szabo D (2018) Finafloxacin: A Novel Fluoroquinolone Introduced in Clinical Trials. Int J Clin Med Microbiol 3: 135. doi: https://doi. org/10.15344/2456-4028/2018/135

Page of

Altogether seven clinical trials have been performed to analyse antibacterial efficacy and toxicity of finafloxacin. A double-blind, placebo controlled, radomized dose escalation study examined toxicity and urinary antibacterial activity of dose of $200 \mathrm{mg}$ and $800 \mathrm{mg}$ finafloxacin in 6 healthy individuals. Drug proved to be safe and tolerable and did't cause serious side effects during the treatment period and urinary recovery was $32.1 \pm 12.2 \%$ and $33.4 \pm 7 \%$, respectively [19].

In a double-blind, placebo controlled, randomized study 95 healthy volunteers were given orally placebo and finafloxacin once daily $(25,50,100,200,400$ and $800 \mathrm{mg}$ dose) or for 7 days $(150,300,600$, $800 \mathrm{mg}$ dose). Headache, diarrhea, loose stool, nause, flatulance, nasopharingitis and rhinitis were the most frequently adverse effects in addition ECG was normal and prolonged QT interval was not found [18].

Pharmacokinetic properties of finafloxacin were evaluated and during adminsitration of once daily dose $(25,50,100,200,400$ and $800 \mathrm{mg}$ ) the $\mathrm{C}_{\text {max }}$ values ranged between $0.24-11.1 \mathrm{mg} / \mathrm{L}$ and $\mathrm{t}_{\max }$ was between 0.5-1 hour, while half life time was 1,28-10 hours [18].

In two studies four drops from $0,3 \%$ finafloxacin was administrated twice daily for 1 week to patients with acut otitis externa caused by $S$. aureus or P. aeruginosa. Clinical cure rates were $71 \%$ and finafloxacin demonstrated high microbiology eradication rate (89\%) against two observed patogens. In two phase III clinical trials studies 618 patients with otitis externa received $0.3 \%$ finafloxacin in otic formulation. Ear pruritus and nause were the most common adverse effect that was present in $1 \%$ of patients [17].

Based on the studies so far, it can be assumed, that finafloxacin is a potential antibacterial agent against major Gram-positive and Gramnegative human pathogens. Moreover, finafloxacin can inhibit biofilm formation of $E$. coli [13], that can be benefitial in therapy of catheter associated infections. Finafloxacin can achieve potency against bacterial pathogens in acidic environment thus, it enables finafloxacin for therapy of $H$. pylori infections.

\section{Competing Interests}

The authors declare that they have no competing interests.

\section{References}

1. Ball P (2000) Quinolone generations: natural history or natural selection? J Antimicrob Chemother 46: 17-24.

2. Paton JH, Reeves DS (1988) Fluoroquinolone antibiotics. Microbiology, pharmacokinetics and clinical use. Drugs 36: 193-228.

3. Stein GE, Goldstein EJ (2006) Fluoroquinolones and anaerobes. Clin Infect Dis 42: 1598-1607.

4. Wright DH, Brown GH, Peterson ML, Rotschafer JC (2000) Application of fluoroquinolone pharmacodynamics. J Antimicrob Chemother 46: 669-683.

5. Bambeke VF, Tulkens PM (2009) Safety profile of the respiratory fluoroquinolone moxifloxacin: comparison with other fluoroquinolones and other antibacterial classes. Drug Saf 32: 359-378.

6. Drlica K, Zhao X (1997) DNA gyrase, topoisomerase IV, and the 4-quinolones. Microbiol Mol Biol Rev 61: 377-392.

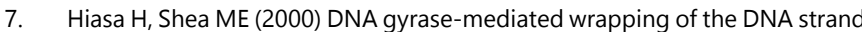
is required for the replication fork arrest by the DNA gyrase-quinoloneDNA ternary complex. J Biol Chem 275: 34780-34786.
8. Aldred KJ, Kerns RJ, Osheroff N (2014) Mechanism of quinolone action and resistance. Biochemistry 53: 1565-1574.

9. Grayson ML, Crowe SM, McCarthy JS, Mills J, Mouton JW, et al. Kucers' The Use of Antibiotics Sixth Edition: A Clinical Review of Antibacterial, Antifungal and Antiviral Drugs. CRC Press.

10. The Sanford Guide To Antimicrobial Therapy (2017) Antimicrobial Therapy Inc. Sperryville, VA, USA.

11. Dalhoff A, Stubbings W, Schubert S (2011) Comparative in vitro activities of the novel antibacterial finafloxacin against selected Gram-positive and Gram-negative bacteria tested in Mueller-Hinton broth and synthetic urine. Antimicrob Agents Chemother 55: 1814-1818.

12. Stubbings $W$, Leow $P$, Yong GC, Goh F, Korber-Irrgang B, et al. (2011) In vitro spectrum of activity of finafloxacin, a novel, $\mathrm{pH}$-activated fluoroquinolone, under standard and acidic conditions. Antimicrob Agents Chemother 55: 4394-4397.

13. McKeage K (2015) Finafloxacin: first global approval. Drugs 75: 687-693.

14. Lemaire S, Bambeke VF, Tulkens PM (2011) Activity of finafloxacin, a novel fluoroquinolone with increased activity at acid $\mathrm{pH}$, towards extracellular and intracellular Staphylococcus aureus, Listeria monocytogenes and Legionellapneumophila. Int J Antimicrob Agents 38: 52-59.

15. Flores-Mireles AL, Walker JN, Caparon M, Hultgren SJ (2015) Urinary tract infections: epidemiology, mechanisms of infection and treatment options. Nat Rev Microbiol 13: 269-284.

16. Barnes KB, Hamblin KA, Richards MI, Laws TR, Vente A, et al. (2017) Demonstrating the Protective Efficacy of the Novel Fluoroquinolone Finafloxacin against an Inhalational Exposure to Burkholderia pseudomallei. Antimicrob Agents Chemother 61: e00082-17.

17. XTORO: HIGHLIGHTS OF PRESCRIBING INFORMATION. These highlights do not include all of the information needed to use XTORO safety and effectively. See full prescribing information for XTORO*.

18. Patel $H$, Andresen A, Vente A, Heilmann HD, Stubbings $W$, et al. (2011) Human pharmacokinetics and safety profile of finafloxacin, a new fluoroquinolone antibiotic, in healthy volunteers. Antimicrob Agents Chemother 55: 4386-4393.

19. Wagenlehner FM, Wagenlehner CM, Blenk B, Blenk $H$, Schubert $S$, et al (2011) Urinary pharmacokinetics and bactericidal activity of finafloxacin (200 and $800 \mathrm{mg}$ ) in healthy volunteers receiving a single oral dose. Chemotherapy 57: 97-107.

20. Genzel GH, Stubbings W, Stîngu CS, Labischinski H, Schaumann R, e al (2014) Activity of the investigational fluoroquinolone finafloxacin and seven other antimicrobial agents against 114 obligately anaerobic bacteria. Int J Antimicrob Agents 44: 420-423. 